\title{
RESULTADOS PRELIMINARES DE PROTOCOLO FITOTERÁPICO PARA A HEMATÚRIA ENZOÓTICA DOS BOVINOS CAUSADA PELA SAMAMBAIA.
}

\author{
Wilmar Sachetin Marçal \\ Universidades Estadual de Londrina - UEL, curso de Medicina Veterinária, Londrina, PR. E-mail: \\ wilmarmarcal@gmail.com
}

\begin{abstract}
RESUMO
A Hematúria Enzoótica Bovina é enfermidade decorrente da ingestão da samambaia (Pteridum arachnoideum), acometendo bovinos em vários estados do Brasil. Devido aos prejuízos econômicos e ao risco à saúde pública, as pesquisas continuam buscando formas de controle da enfermidade, já que existem locais que ainda se criam bovinos em contato direto com a planta, principalmente no estado do Paraná, onde a topografia é bem acidentada, sem possibilidade de mecanização da terra para correção da acidez do solo. Num levantamento em municípios paranaenses, detentores da samambaia em suas pastagens, o autor correlaciou a existência da planta à manifestação natural de Hematúria Enzoótica dos Bovinos. Foram utilizados fitoterápicos para combater a enfermidade, com resultados preliminares promissores. Além disso, o protocolo proposto permitiu, também, minimizar prejuízos aos pecuaristas, com orientações educativas sobre os riscos de consumir os subprodutos de origem animal, como carne e leite.
\end{abstract}

Palavras-chave: enfermidade, samambaia, saúde pública, vacas.

\section{PRELIMINARY RESULTS OF PHYTOTHERAPEUTIC PROTOCOL FOR BOVINE ENZOOTIC HEMATURIA CAUSED BY BRACKEN FERN.}

\begin{abstract}
Enzootic Bovine Hematuria is a disease caused by the ingestion of the bracken fern (Pteridum arachnoideum), affecting cattle in several Brazilian states. Due to economic losses and the risk to public health, research continues to seek ways to control the disease, since there are still places where cattle are still kept in direct contact with the plant, mainly in the state of Paraná, where the topography is very uneven, without the possibility of mechanization of the soil to correct soil acidity. In a survey carried out in Paraná cities, bracken fern holders in their pastures, the author correlated the existence of the plant with the natural manifestation of Bovine Enzootic Hematuria. Phytotherapies were used to combat the disease, with promising preliminary results. In addition, the proposed protocol also allowed to minimize losses to cattle ranchers, with educational guidelines on the risks of consuming animal by-products such as meat and milk.
\end{abstract}

Keywords: diseases, bracken fern, public health, cows.

\section{INTRODUÇÃO}

O Estado do Paraná almeja alcançar o pico de sua produção agropecuária, por deter eficiente tecnologia e indiscutível produtividade (MARÇAL 2003), com um efetivo bovino de 9.413.937 cabeças (IBGE, 2012). Entretanto, demonstra alguns problemas na criação animal, apresentando graves enfermidades relacionadas à ingestão de vegetais tóxicos (CARVALHO et al., 2011). Nesse enfoque, destaca-se a Pteridium arachnoideum, planta tóxica popularmente conhecida por samambaia dos campos, ou simplesmente samambaia. Trata-se de um vegetal de 
característica invasora, frequente em solos ácidos, arenosos e de baixa fertilidade. É uma planta perene, rizomatosa, herbácea, ereta e ramificada, medindo entre 50 a $180 \mathrm{~cm}$ de altura. Infesta campos, matas ciliares, capoeiras, beiras de matos e de estradas.

Segundo Hojo-Souza et al. (2010), a samambaia encontra-se ainda mais disseminada em função da destruição dos ecossistemas naturais, que foram sendo transformados em extensas áreas agropastoris e agrícolas para atender a demanda da humanidade. Neste contexto, segundo várias informações da mídia brasileira e mundial, áreas florestais que serviam de reserva natural, sofreram desmatamento, destoca e abrigam pastagens, sem, contudo,ocorrer correção do solo (MARÇAL, 2003).

A Pteridium arachnoideum tem sido considerada a planta tóxica mais representativa aos prejuízos da pecuária bovina paranaense, ocorrendo em 105 municípios (POLACK, 1990), dos 370 estudados (FERREIRA, 1996). Relatos de surtos e de ocorrência de problemas com a samambaia afetando rebanhos de bovinos no estado do Paraná já foram observados por Basile et al. (1981), Polack (1990), Niero et al. (1991), Oliveira et al. (1998), Marçal et al. (2001) e Carvalho et al. (2011).

Embora a planta não seja palatável, determinadas condições favorecem sua ingestão pelos bovinos. Em épocas de escassez alimentar, a fome constitui a primeira causa básica de ingestão da samambaia pelos bovinos. Isto normalmente ocorre na estação seca (julho a outubro), pois a planta suporta bem o período sem chuvas, possibilitando sua procura pelos animais. Os animais também podem ingerir os brotos da samambaia, quando houver escassez de pastagens devido à seca, geada, queimada ou mesmo pela superlotação (PARKER, 1965).

Segundo estudiosos, os bovinos que ingerem a samambaia acabam "viciados na planta", podendo permanecer consumindo mesmo tendo acesso à pastagem natural (TOKARNIA et al., 1979), caracterizando, com isso, ingestões repetidas e compulsivas. Outro fator que possibilita que os animais procurem a planta é a carência de pastagem fibrosa. Como a samambaia costuma se desenvolver e atingir boa altura, os bovinos suprem a necessidade de fibra, comendo os caules e folhas longas que a planta normalmente possui.

A forma de manifestação da toxidez conhecida por Hematúria Enzoótica dos Bovinos, possui como principais características a evolução crônica, com hematúria intermitente e emaciação. Ocorre perda de sangue, sem reposição pela medula (anemia aplástica), acometendo vacas prenhes e causando aborto (MARÇAL et al., 2001; CARVALHO et al., 2011).

A Pteridium arachnoideum é também tóxica ao homem (TOKARNIA et al., 1979; LORENZI, 1982). Na Europa, o consumo de samambaia como alimento humano foi muito utilizado em épocas de deficiência alimentar, como por exemplo, durante a Primeira Guerra Mundial (ULIAN et al., 2010). No Japão há um prato típico conhecido por "warabi" que consiste em brotos de samambaia servidos como salada para seres humanos (MARÇAL, 2003). De acordo com Marliére et al (1998), no estado de Minas Gerais, em Ouro Preto, a população tem o hábito de consumir os brotos de samambaia. A alta incidência de câncer de esôfago e estômago na região, em relação a outras áreas do estado, motivou os autores a investigar a possível influência da ingestão do vegetal na ocorrência daquelas patologias. O consumo de broto de samambaia foi identificado através de entrevista com os pacientes próximos, em caso de morte do paciente. Os autores concluíram neste estudo epidemiológico que houve uma "Odds Ratio", ou seja, razão de chances, fortemente elevada para tumores de esôfago e estômago em pessoas que ingeriram o broto da samambaia.

Sabendo-se da importância da Hematúria Enzoótica dos Bovinos como impactante negativa na saúde e bem-estar animal, além dos prejuízos econômicos que causam aos pecuaristas e a interface com a saúde públia, o presente trabalho objetiva demonstrar resultados preliminares Colloquium Agrariae, vol. 13, n. Especial, Jul-Dez, 2017, p. 242-248. ISSN: 1809-8215. DOI: 10.5747/ca.2017.v13.nesp.000200 
sobre a fitoterapia em bovinos naturalmente criados numa região endêmica, onde há presença significativa da Pteridium arachnoideum.

\section{METODOLOGIA}

As propriedades rurais selecionadas situavam-se no município de Ortigueira, região norte do estado do Paraná (Situado a 818 metros de altitude, Latitude: $24^{\circ} 13^{\prime} 30^{\prime \prime}$ Sul e Longitude: $50^{\circ}$ 55' 42" Oeste). O município possui uma topografia fortemente ondulada, com solo podzólico vermelho escuro e vermelho amarelado, cambissolo e solos litólicos.

A pesquisa que originou o presente trabalho recebeu aprovação do Comitê de Ética no Uso de Animais (CEUA) da Universdade Estadual de Londrina (UEL), através dos processos de números 9004.2015.89 e 12209.2015.38. Nesse estudo, foram trabalhadas 14 (quatorze) vacas não gestantes, pertencentes a duas fazendas selecionadas, na qual exista infestação da samambaia. Os bovinos foram divididos em dois tratamentos: Testemunha (T1) composto por 07 vacas sem hematúria e o grupo das vacas tratadas (T2), igualmente composta por 07 vacas enfermas, ou seja, com manifestação natural de Hematúria Enzoótica causada pela planta Pteridium arachnoideum. Os bovinos do Tratamento T2 receberam, por via oral, 60 (sessenta) $\mathrm{ml}$ do composto NUR4C pulverizado em $20 \mathrm{~kg}$ de sal mineral pronto, servido no cocho durante o primeiro e o décimo dia do experimento. Depois, numa segunda etapa, ou seja, no décimo primeiro dia e vigésimo dia do experimento, as vacas receberam, também, por via oral misturado no sal mineral, 60 (sessenta) $\mathrm{ml}$ do composto NUR5C, igualmente pulverizado em $20 \mathrm{~kg}$ de sal mineral pronto, fornecido no respectivo cocho. Conforme a preparação, os compostos NUR4C e NUR5C eram fitoterápicos naturais, extratos de nosódio urinário e aquoso da planta Pteridium arachnoideum, com diferentes concentrações, desenvolvidas para o tratamento proposto. Durante a realização da presente alternativa terapêutica, os bovinos permaneceram em área de pastagem, com água a vontade e sem a presença de samambaia.

As vacas foram avaliadas quanto à condição corporal (com escala de magra, moderada ou gorda) e quanto ao tipo de manifestação da hematúria (contínua ou intermitente). As avaliações foram semanais e mensais, de acordo com os protocolos propostos.

Foram realizadas análises de urina das 07 vacas enfermas do tratamento $\mathrm{T} 2$, sendo as amostras colhidas através da indução vulvar manual e acondicionadas em recipientes próprios para urinálise. Após a colheita, as amostras foram mantidas em refrigeração até o destino final no laboratório. A urinálise foi realizada através de fita reagente (Hemagen ${ }^{\circledR}$ ), mensuração da densidade no refratômetro específico e pela sedimentoscopia, conforme apregoam Medeiros (1993) e Navarro (1996). As amostras de urina foram analisadas antes e ao término dos protocolos experimentais.

Os dados obtidos foram avaliados através do programa SAEG (UFV, 2007). Os resultados foram avaliados por testes qualitativos não paramétricos. O teste empregado para a comparação de dados qualitativos foi o Teste do Qui-Quadrado (Chi-Square), representado por $\mathrm{X}^{2}$. Isso permitiu testar a diferença entre duas proporções e verificação da independência de dois fatores, além de ser utilizado para comparação de mais de dois grupos.

\section{RESULTADOS}

Os resultados observados no presente ensaio encontram-se destacados abaixo, distribuídos em tabelas e figura gráfica. A tabela 1 apresenta os resultados das urinálises, antes e após o tratamento fitoterápico em 7 vacas experimentais enfermas. 
Tabela 1: Valores de hemácias encontradas na urinálise

\begin{tabular}{ccc}
\hline Número da vaca & Antes do fitoterápico & Depois do fitoterápico \\
\hline 01 A & $3.800 / c$ & $460 / \mathrm{c}$ \\
02 A & $2.500 / \mathrm{c}$ & $2 / \mathrm{c}$ \\
03 A & $4.500 / \mathrm{c}$ & $110.400 / \mathrm{c}$ \\
04 A & $3.900 / \mathrm{c}$ & $1250 / \mathrm{c}$ \\
05 A & $250 / \mathrm{c}$ & $0 / \mathrm{c}$ \\
06 A & $3 / \mathrm{c}$ & $0 / \mathrm{c}$ \\
07 A & $3.200 / \mathrm{c}$ & $4.000 / \mathrm{c}$ \\
\hline
\end{tabular}

Fonte: $O$ autor, 2017.

Tabela 2 - Estatísticas dos dados gerais.

\begin{tabular}{ccccc}
\hline $\begin{array}{c}\text { Média das } \\
\text { Hemácias }\end{array}$ & \multicolumn{2}{c}{ Mediana Variância } & $\begin{array}{c}\text { Desvio } \\
\text { Padrão }\end{array}$ & $\begin{array}{c}\text { Coeficiente de } \\
\text { Variação \% }\end{array}$ \\
\hline $1.613,75 / c$ & 855 & 2.975 .122 & $1.724,854$ & 106,88 \\
\hline
\end{tabular}

Fonte: 0 autor, 2017.

Tabela 3 - Estatísticas aplicadas por grupos.

\begin{tabular}{ccc}
\hline & $\begin{array}{c}\text { Antes dos Compostos } \\
\text { Fitoterápicos }\end{array}$ & $\begin{array}{c}\text { Depois dos Compostos } \\
\text { Fitoterápicos }\end{array}$ \\
\hline Média das hemácias & $2.275,50 / c$ & $952 / \mathrm{c}$ \\
Mediana & 2.850 & 231 \\
Variância & 3.027 .022 & 2.467 .256 \\
Desvio Padrão & $1.739,834$ & $1.570,75$ \\
Coeficiente de variação \% & 76,4594 & 164,9948 \\
\hline
\end{tabular}

Fonte: 0 autor, 2017.

Observou-se maior coeficiente de variação das observações para Depois dos compostos, em comparação a Antes do tratamento.

Na figura 1, logo abaiixo, é possível observar que ainda há um outlier no tratamento depois dos compostos. $\mathrm{O}$ animal identificado como $07 \mathrm{~A}$, foi o único que teve o valor aumentado de hemácias depois do tratamento composto, provavelmente por outro fator intercorrente contaminante durante o experimento. Os demais parâmetros da urinálise deste após o tratamento também se encontram alterados, como $\mathrm{pH}$ antes $=6,0 / \mathrm{pH}$ depois $=9,0$; proteínas antes $=++/$ proteínas depois $=+++$; sangue antes $=++/$ sangue depois $=++++$; leucócitos antes $=06 \mathrm{c} /$ leucócitos depois = $12 \mathrm{c}$; bactérias antes $=$ negativo $/$ bactérias depois $=++$. Para a continuação da análise estatística, decidiu-se que as observações do animal $07 \mathrm{~A}$ permanecessem no conjunto de dados da experimentação científica.

Considerando que pressupostos não foram atendidos, aplicou-se o Teste T para 2 amostras Colloquium Agrariae, vol. 13, n. Especial, Jul-Dez, 2017, p. 242-248. ISSN: 1809-8215. DOI: 10.5747/ca.2017.v13.nesp.000200 
dependentes com variâncias homogêneas, calculando-se o $p$-value= 0,1164. Como este valor foi superior a 0,05, aceitou-se a hipótese nula que as médias de hemácias dos tratamentos antes e depois são iguais.

Figura 1 - Gráfico tipo Boxplot - Hemácias versus tratamento.

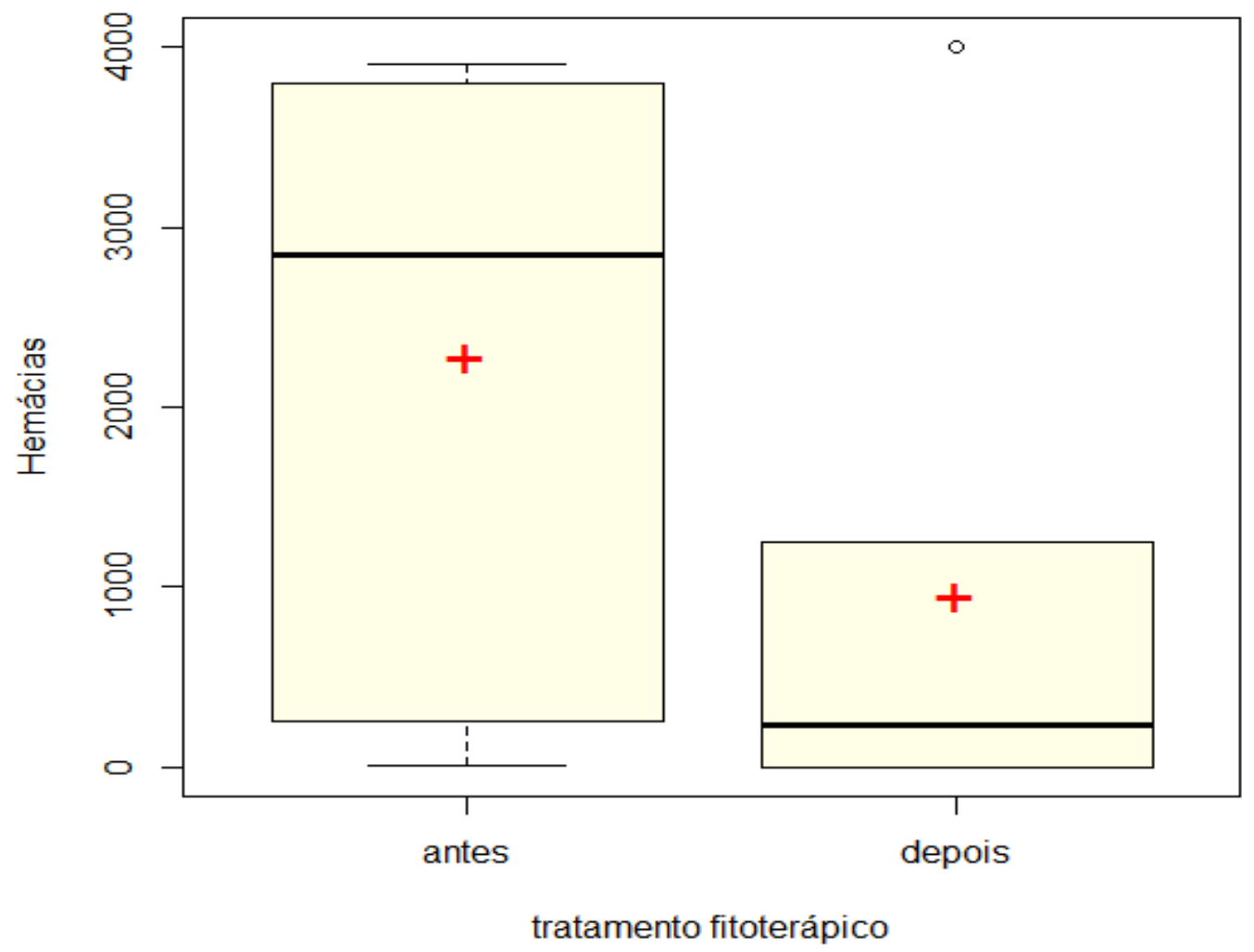

\section{DISCUSSÃO}

A correlação direta da existência da Pteridium arachnoideum, popularmente conhecida por samambaia, em propriedades rurais e o aparecimento de Hematúria Enzoótica em Bovinos está bem consolidada pelos estudiosos em todo o mundo, incluindo o Brasil, conforme alertaram Tokarnia et al. (1979); Oliveira et al (1998) e Marçal (2003). Essa situação é facilmente detectada em munocípios onde a planta ainda representa muitos prejuízos a ecuária bvina, como é o caso de Ortigueira, no estado do Paraná.

Sabendo do alto grau de morbidade e consequente letalidade causado pelo consumo de samambaia e a ausência de tratamento efetivo, a pesquisa com fitoterápicos mostrou-se com certo potencial de melhora clínica do animal. Apesar de não ser a cura efetiva para essa doença, o tratamento auxiliou na recuperação do animal, o que garantiu a melhora do bem estar e evitou a perda e os prejuízos econômicos ao produtor. Tal prospecção abre novas perspectivas para a mitigaão dos efeitos dessa planta à saúde animal, em particular de vacas de cria, conforme observado também por Oliveira et al (1998) e Marçal (2003).

\section{CONCLUSÕES}

Com 95\% de nível de confiança, não houve evidências estatísticas que comprovam que o tratamento com os compostos provocou redução na média das hemácias na urina.

Colloquium Agrariae, vol. 13, n. Especial, Jul-Dez, 2017, p. 242-248. ISSN: 1809-8215. DOI: 10.5747/ca.2017.v13.nesp.000200 
Sugere-se a repetição do experimento com um número maior de repetições, visto que há indicativos clínicos que comprovem a eficácia do tratamento homeopático e fitoterápico propostos, pois 5 animais tiveram redução da contagem de hemácias na urinais. Além disso, clinicamente houve melhora no escore corporal dos bovinos e preservação de sinais clínicos importantes, como locomoção, busca pelo sal mineral, pastoreio e ingestão voluntária de água.

\section{REFERÊNCIAS BIBLIOGRÁFICAS}

BASILE, J. R.; GASTE, L.; REIS, A.C.F. Intoxicação aguda de bovinos pela samambaia (Pteridium aquilinum) no estado do Paraná. Revista de Ciências Agrárias, Curitiba, v.3, p.167-170, 1981.

CARVALHO, M. C.; MARÇAL, W.S.; BALARIN, M. R.; FORTES, M. S.; PARIZOTTO JUNIOR, N.; CAMARGO, M.I. Avaliação bioquímica do ácido siálico como biomarcador tumoral em novilhas criadas em propriedade endêmica para hematúria enzoótica dos bovinos. Ciência Animal Brasileira, v.12, n.2, p.306-310, 2011. https://doi.org/10.5216/cab.v12i2.3431

FERREIRA, J. C. V. O Paraná e seus municípios. Maringá: Memória Brasileira, 1996. p.569-570.

HOJO-SOUZA, N. S.; CARNEIRO, C. M.; SANTOS, R. C. Pteridium aquilinum: o que sabemos e o que ainda falta saber. Bioscience Journal, v.26, n. 5, p.798-808, 2010.

IGBE. Produção da Pecuária Municipal, 2012. Instituto Brasileiro de Geografia e Estatística. Disponível

em: ftp://ftp.ibge.gov.br/Producao Pecuaria/Producao da Pecuaria Municipal/2012/ppm2012.p df. Acesso em 29 de setembro de 2015.

LORENZI, H. Pteridium aquilinum (L) Kuhn. In: Plantas daninhas do Brasil: terrestres, aquáticas, tóxicas e medicinais. Nova Odessa, p.341, 1982.

MARÇAL, W. S.; GASTE, L.; REICHERT NETTO, N. C.; GARGANTINI, M.; FERNANDES, R. P.; MONTEIRO, A. A. Ocorrência de intoxicação aguda em bovinos pela samambaia (Pteridium aquilinum, L. Kuhn) no norte do Paraná - Brasil. Semina, Londrina, v.22, n.2, p.139144, jul./dez. 2001.

MARÇAL, W. S. A intoxicação por samambaia em bovinos criados no Estado do Paraná. Semina, Londrina, v. 24 , n. 1 , p. 197-208, jan./jun. 2003. https://doi.org/10.5433/1679$\underline{0359.2003 v 24 n 1 p 197}$

MARLIÉRE, Cláudia A; SANTOS, Rinaldo C; GALVÄO, Márcio A. M; SOARES, José F; EVANGELISTA, Cynara L. M; GOMES, Roberto Q. F. Ingestão de broto de samambaia e risco de câncer de esôfago e estômago na região de Ouro Preto, MG. Rev. Bras. Cancerol; 44(3):225-9, jul.-set. 1998.

MEDEIROS, A. S. Semiologia Urológica, Rio de Janeiro: Medsi, pág. 73-93, 1993.

NAVARRO, C. E. K., Manual de Urinálise Veterinária, São Paulo: Varela, pág. 89, 1996.

NIERO, L.; MARÇAL, W. S.; REIS, A.C.F.; ACCORSI, E. Surto de intoxicação aguda em bovinos pela Colloquium Agrariae, vol. 13, n. Especial, Jul-Dez, 2017, p. 242-248. ISSN: 1809-8215. DOI: 10.5747/ca.2017.v13.nesp.000200 
ingestão de samambaia (Pteridium aquilinum, L. Kuhn) no norte do Paraná. In: 9o Simpósio de Estagiários do CCB/UEL, 1991, Anais. Londrina. UEL, 1991. p.90.

OLIVEIRA, G. P. MATSUMOTO, T.; PRIMAVESI, A. C. Ocorrência de intoxicação causada por samambaia (Pteridium aquilinum) na região nordeste do Paraná. Comunicado Técnico Embrapa, São Carlos, n. 20, p.1-9, out. 1998.

PARKER, W. H. \& McCREA, C. T. Bracken (Pteris aquilina)poisoning of sheep in the Nort York Moors. The Veterinary Record, 77: 861-866,1965.

POLACK, E.W. Toxicidade da Pteridium aquilinum no Estado do Paraná. 1990. Dissertação (Mestrado) - Universidade Federal do Paraná, Curitiba. 1990.

SAEG. Sistema para Análises Estatísticas, Versão 9.1: Fundação Arthur Bernardes - UFV - Viçosa, 2007.

TOKARNIA, C. H.; DOBEREINER, J.; BARROS, S. S. Plantas tóxicas do Brasil. Região Sul. Rio de Janeiro: Universidade Federal Rural do Rio de Janeiro, 1979.

ULIAN, C. M. V.; BAPTISTA, A.A.S.; VENTURA, R.F.A.; SAKATE, M. Pteridium aquilinum na alimentação humana: uma revisão. Acta Veterinaria Brasilica, v.4, n.2, p.64-69, 2010.

Ceua UEL protocolos 9004.2015.89 e 12209.2015.38 